\title{
A Data Classification Algorithm of Internet of Things Based on Neural Network
}

\author{
https://doi.org/10.3991/ijoe.v13i09.7587 \\ Zhenjun Li \\ Hunan Radio and TV University, Hunan, China \\ $278060389 @$ qq. com
}

\begin{abstract}
To alleviate the pressure of data size, data transmission and data processing in the huge data dimension of the Internet of things., data classification is realized based on back propagation (BP) neural network algorithm. The working principle is deduced in detail. For the shortcomings of slow convergence and easy to fall into the local minimum, the combination of variable learning and momentum factors is used to improve the traditional back propagation algorithm. The results show that the optimized algorithm improves the convergence speed of the network to a certain extent. Therefore, it is concluded that the back propagation neural network has higher classification success rate when classifying multidimensional data in Internet of things.
\end{abstract}

Keywords-Internet of things, data classification, BP neural network, algorithm research

\section{$1 \quad$ Introduction}

The main functions of the Internet of Things are information identification, intelligent control, information identification and location monitoring. From a business perspective, they belong to the vertical application model, the public service model and the industry common platform model [1]. Vertical application is for a specific enterprise or industry to carry out business. It meets the needs of some specific business and industry, such as the enterprise applications of electricity, oil, road and rail [2]. The public service model is used in the service industry, such as e-commerce, consulting and public transport management. Industry common model is a class of industry and its related businesses and industries, such as medical-related areas and logistics-related areas [3]. At present, the Internet of things is at the start-up stage of the industry [4]. However, it is developing rapidly, and many applications have been widely used [5]. The application of Internet of things promotes the development of social productive forces, and brings great convenience to our daily life and work. The process of data classification is an analysis of an existing data set with many types. Finally, it will extract the same type of data features, and then classify the new unknown data based on the extracted data features. So far, great achievements have been made in the development of classification techniques, and various classification techniques and theories have been put forward. We can divide them into three broad cate- 
gories. The first is the use of statistical principles, such as vector machines, regression models, Bayesian networks and other methods. The second is the use of the principle of connection, such as the proposed artificial neural network algorithm. The third kind is the classification of some specific rules, such as rough set theory, association rules and decision tree [6].

\section{State of the art}

So far, many researchers have made great achievements in the field of data classification. Among them, it includes the Bias classification and the decision tree classification. After that, the researchers found that feedforward neural networks can be used in data classification. At the same time, it has the advantages of high classification accuracy, strong anti-interference ability and fast classification. The feedforward neural network is quickly applied to data mining data classification. At present, although we cannot fully explain all the reasons for the learning and data classification of feedforward neural networks, the super generalization and learning ability allow feed-forward neural networks to quickly learn the raw data rules [7]. In practical applications, some data warehouse software providers have begun to develop data classification tools based on the principle of feed forward neural networks. Neural network is a complex network system composed of many simple neurons, and they are widely connected with each other. Its design philosophy stems from the organizational structure of the human brain. Therefore, it has many features of human brain function, and can simulate human brain activity simply. With the rapid development of Internet of things and the maturity of cloud computing technology, the application of feed-forward neural network to the Internet of things with large amounts of data has become a new research hotspot. In addition, the classification of high-dimensional data is also an important and challenging topic in itself.

The main contents of this paper are to realize the data classification function in the environment of Internet of things using BP neural network. In the practical application, it is found that the traditional BP neural network has the problems of slow convergence and easy to fall into the local minimum. Obviously, these problems will restrict the application of BP neural networks in the Internet of things with large amounts of data. In order to verify the effectiveness of the improved algorithm, a data classification experiment system is implemented in the MATLAB environment by making full use of the neural network toolkit and the GUI interface. The system is used to classify the data collected by the coal mine safety monitoring system, and the network convergence performance is compared before and after the improved algorithm. 


\section{$3 \quad$ Methodology}

\subsection{The basic principle of BP neural network}

Among the various feedforward neural networks, the widely used training algorithm is the error back propagation algorithm, that is, the BP algorithm. In general, feedforward neural networks that use the BP algorithm for training are called BP neural networks. In the feedforward neural network learning problem, the BP algorithm has good performance. According to statistics, in the actual application of artificial neural networks, $80 \%$ to $90 \%$ of the artificial neural network model uses BP network. It is also the core part of the forward network, which embodies the best part of the artificial neural network. The learning process of BP algorithm can be divided into two stages. They are the training input data forward propagation stage and the error signal back propagation stage, as shown in Figure 1.

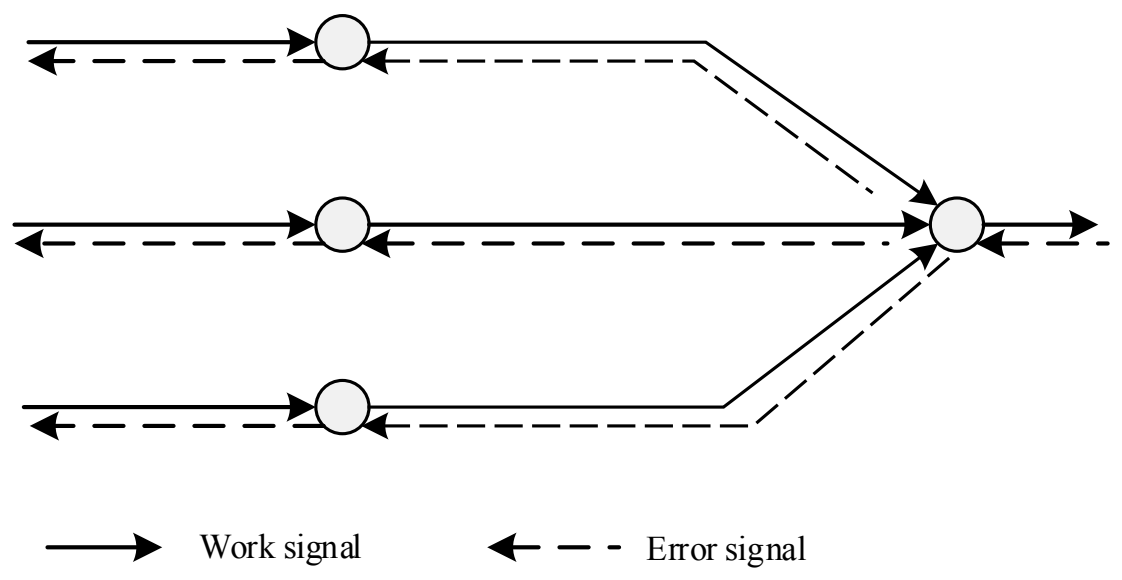

Fig. 1. Signal transmission of BP algorithm

Forward propagation phase of training data. The raw input data is imported from the input layer. Then, it is processed by hidden layer neurons. Finally, the output data is generated by the output layer. In the forward transmission of data (from left to right in the figure), the connection weights between the layers of the BP neural network do not change. The weights of each layer of neurons affect only the subsequent state of the neurons that are connected between the neurons after the data is passed. If the output data is not consistent with the expected prediction, the BP neural network is not fully trained, and then, it shifted to the error signal back propagation stage.

Back propagation stage of error signal. In the reverse phase of the error signal, the error signal from the actual output and the ideal output is transmitted from the output layer to the input layer of the network. When the error signal is transmitted one layer at a time, we modify the weights between the threshold of the previous layer and the current two layers according to certain rules, so that the output results can be approximated to the target output. 


\subsection{Comparison of network training results and algorithm improvement}

Once the data has been normalized, the neural network can be trained. First, the BP neural network structure needs to be selected. According to the structural analysis of the data set, this experiment should choose 10 contacts as the input layer, 6 basis points as the output layer, and the number of hidden layer selection to the current has not yet determined the formula. Different hidden nodes will have an impact on the experimental results. This article first set to 21, that is, about 2 times the input layer. In the later experiment, this paper will compare the convergence speed and classification error of the number of different hidden nodes, so as to determine the optimal network structure. Once the structure of the BP network is initially determined, the network is trained by the input and output sample sets. The threshold and weights of the network are learned and corrected, so that the network implements a given inputoutput mapping relationship.

Before the start of the training, the experimental system also requires the user to enter the target network accuracy and maximum number of training. When the trained BP neural network reaches one of these two conditions, the entire training will be terminated. The network accuracy of this experiment is 0.01 , and the maximum number of training is 10000 times. When the training BP network button is clicked, the system begins to train the configured BP network. The result is shown in Figure 2.

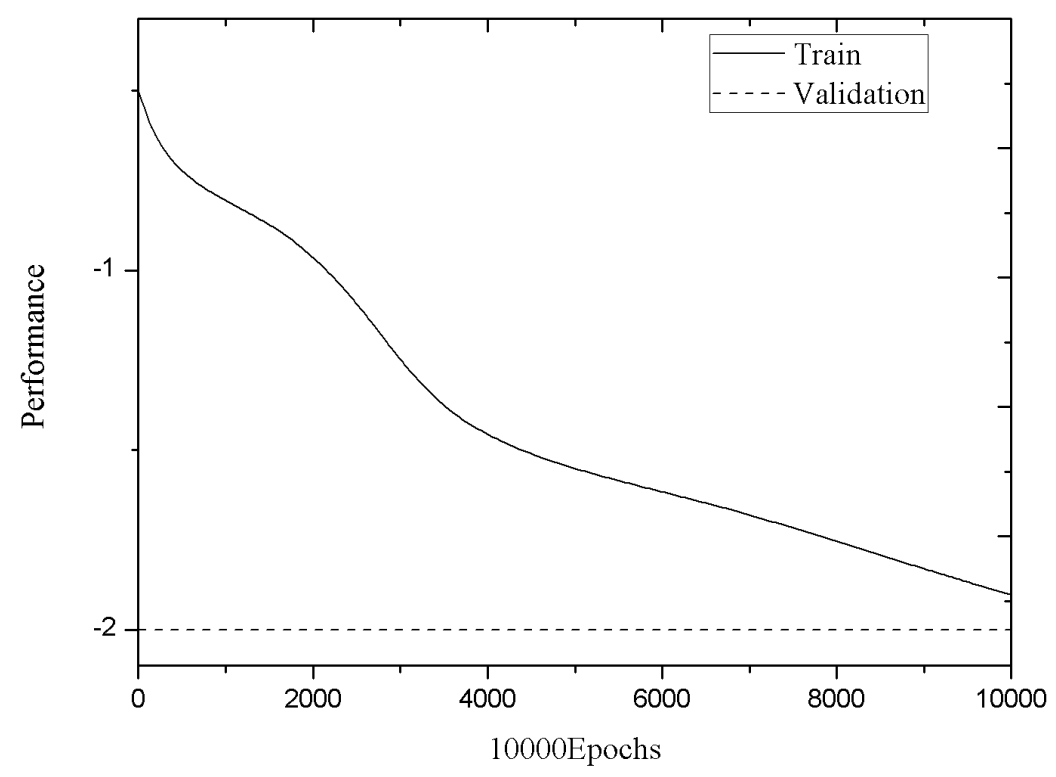

Fig. 2. BP network training convergence graph

According to the convergence graph after the training, it is found that the learning rate of the current BP network is 0.0122305 when the number of training iterations reaches the maximum of 10,000 times, and it is clear that the target network accuracy cannot reach 0.01 . It is found that the network accuracy of training is very small when 
the training times are between 200 and 2500 times. However, after exceeding the number of times, network accuracy has shown a rapid drop phenomenon. Obviously, the training of the network undergoes a weight correction flat area between 200 and 2500 intervals. In this case, the network should speed up the learning step, so as to achieve convergence as soon as possible. In this experiment, through the use of neural network experiment box tool provided by MATLAB, the implementation of the above improved algorithm is completed. In the system interface, first, the trained network action is clicked to select the training options using the improved algorithm. Then, the training BP network button is clicked again, and the system begins to train with the improved BP algorithm. The selected BP network structure and training parameters are consistent with the previous training, and the training results are shown in Figure 3.

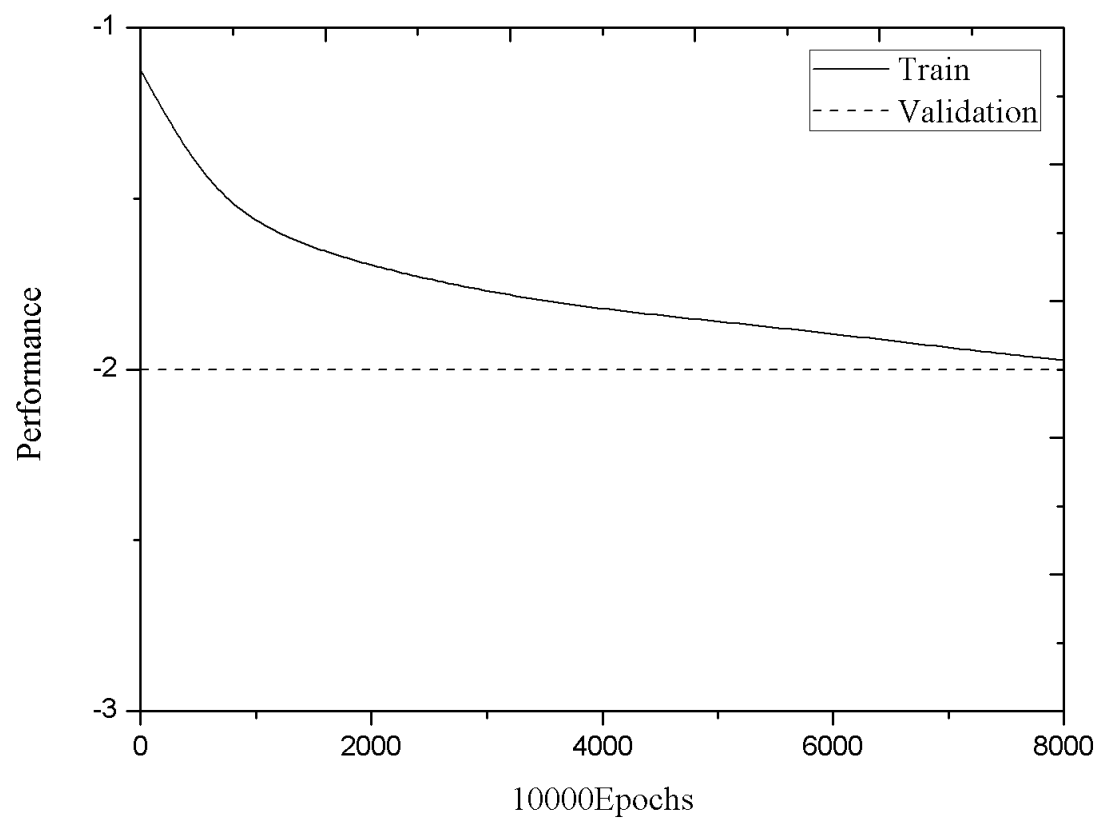

Fig. 3. The training convergence graph of the improved BP algorithm

It is obvious that the training curve of network precision of BP neural network is smoother after adding momentum factor. Moreover, the training results can show that the network precision of BP neural network has reached the target network accuracy by 0.01 when the training iterations reach 8509 times. From the structure of the curve, it can be seen that the change of the weight of the whole training process becomes smaller gradually, and it does not appear to be similar to the relatively gentle interval in the first training. In order to reflect the training situation of BP network comprehensively, this paper uses traditional BP algorithm and improved BP algorithm to train several times. The target network precision is adjusted to 0.001 and the number of iterations is increasing. The final results are shown in Table 1. 
Paper-A Data Classification Algorithm of Internet of Things Based on Neural Network

Table 1. Comparison of network learning rate and training frequency

\begin{tabular}{|l|c|c|c|c|c|c|c|}
\hline Number of network iterations & 100 & 500 & 1000 & 5000 & 10000 & 20000 & 50000 \\
\hline BP algorithm network accuracy & 0.4411 & 0.3212 & 0.1823 & 0.0782 & 0.01223 & 0.00452 & 0.00282 \\
\hline $\begin{array}{l}\text { Improved BP algorithm net- } \\
\text { work accuracy }\end{array}$ & 0.4524 & 0.2721 & 0.1084 & 0.0578 & 0.00892 & 0.00354 & 0.00258 \\
\hline
\end{tabular}

From the final results, it can be seen that the improved algorithm improves the convergence performance of BP neural network to a certain extent. In addition, the two training results show that the BP neural network converges faster at the beginning of training. With the increase of training times, the convergence speed is obviously slow. When the training is more than 50000 times, the accuracy of the network is very small under the two algorithms. In this interval, the convergence speed of the improved algorithm does not show obvious advantages. Thus, when the BP neural network error is reduced to a certain extent, the learning rate must be maintained at a lower value. At this point, excessive increase in learning rate is likely to occur concussion.

\subsection{Data classification and cross validation}

The trained BP network can be used to classify the detected data. In order to better analyze the error, the threshold range of the 6 output contacts is set at $[0,1]$. The final classification result is represented by a combination of 6 output contact values, and the prediction type result depends on the largest one in the final output value. If the output value is $[0.1,0.0,0.9,0.0,0.1,0.0]$, the classification result is type 3 with an error of - 0.1 . To facilitate the presentation of the results, we add a value of 1 less to the predicted result value. The final result is recorded as 2.9. Thus, the original sixdimensional vector becomes one-dimensional, and it can be compared with the actual data type on the coordinate map. In addition, in error analysis, we stipulate that the maximum error shall not exceed 1 . Because the biggest error for both categories is to completely determine the error, so the error is greater than 1 and meaningless. Therefore, if the actual value in the above example is 4 (that is, the error is 1.1 ), then we calculate the error automatically converted into 1 .

In this experiment, we divide the data into two groups. They are training set and test set, and each data set has 1000 samples. In the first experiment, we use the training set to train the neural network, and use the test set to verify the trained network. In order to make the experiment result more accurate and avoid the influence of the uneven distribution of data, this paper adopts cross validation strategy later. That is, in the second experiment, the exchange of two sets of data sets was used to do the same test. 


\section{$4 \quad$ Result analysis and discussion}

\subsection{Determination of the number of hidden layer junctions}

In this experiment, by training BP networks with different hidden layer nodes, they can achieve the same target network accuracy of 0.01 . The network accuracy in training is only the network error for the training sample set, and each time the value is derived from the classification bias of a single sample. Therefore, it cannot represent the whole network performance. Through the error of the classification results and the training times of the target network accuracy, the optimal classification structure suitable for the data set is calculated. In the whole process, the mean square error of the hidden layer from 10 to 40 nodes is MSE. The result is shown in Figure 4.

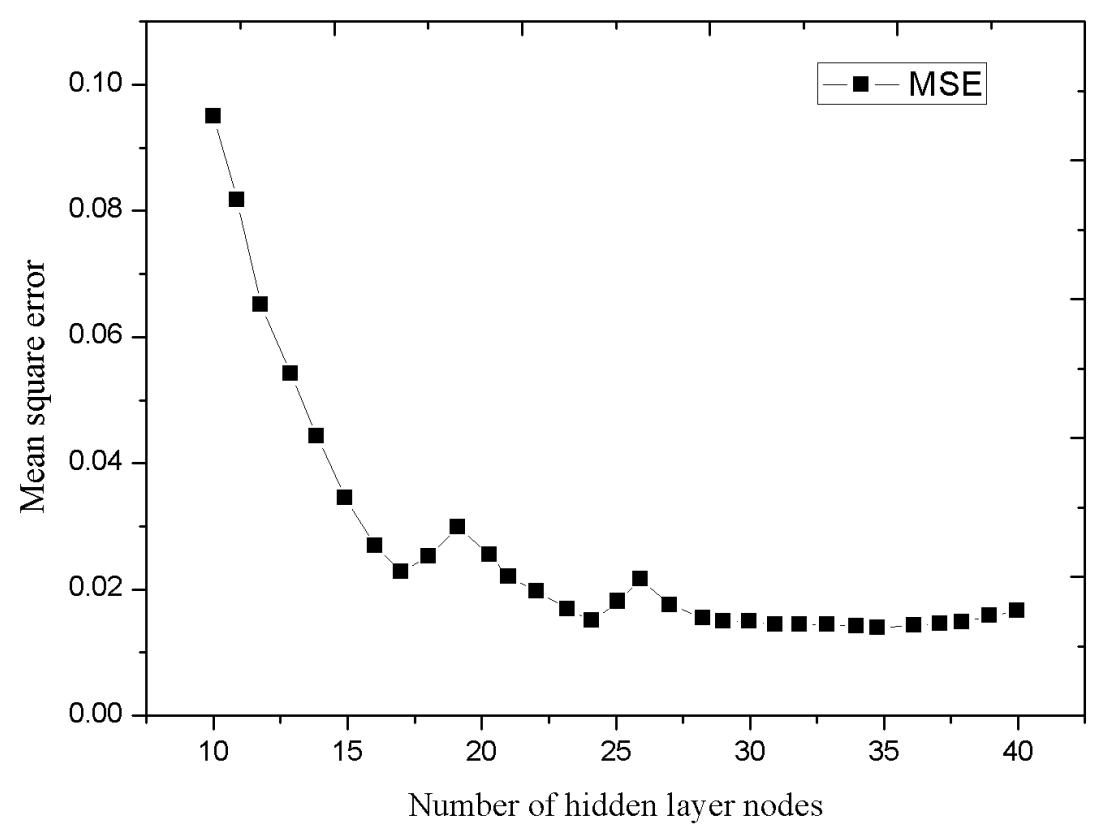

Fig. 4. Relation between number and variance of hidden layer junction

The experimental results show that, within certain range, the increase of hidden layer number of BP neural network can reduce the mean square error of classification. When the BP networks with different hidden layer nodes reach the target network accuracy (0.01), their training times curve is shown in Figure 5. 


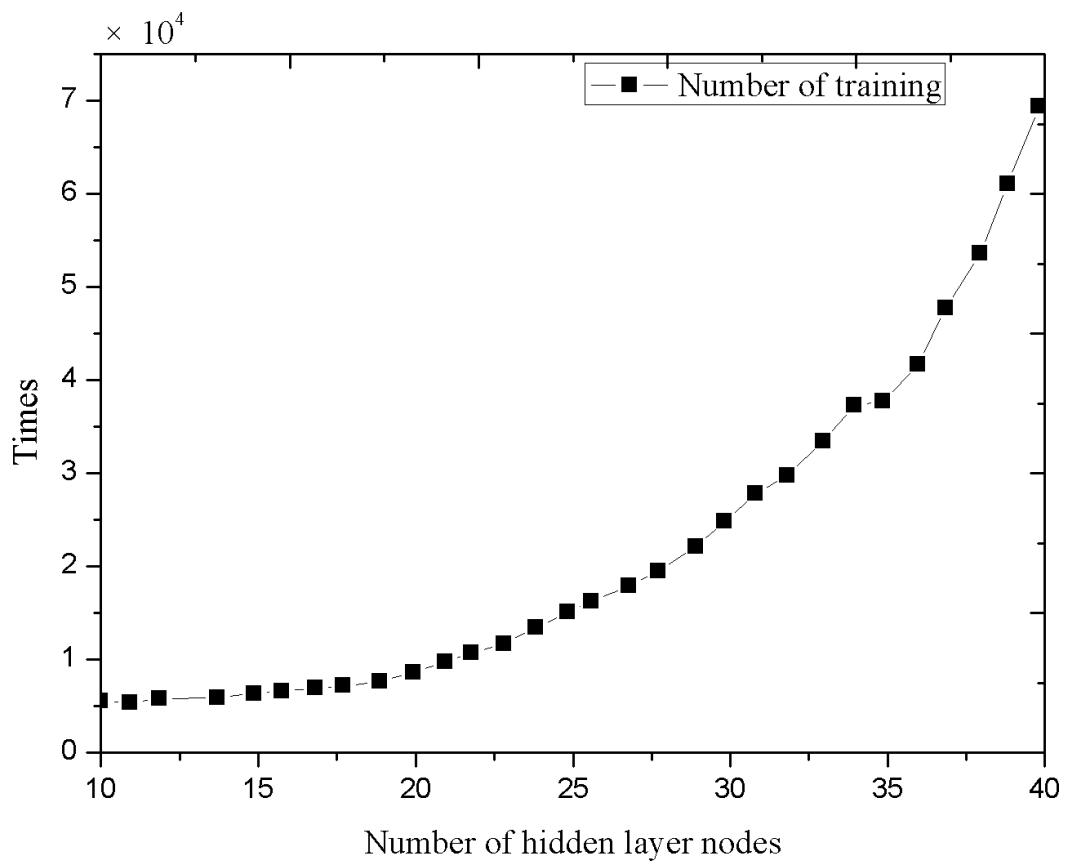

Fig. 5. Relation between the number of hidden layer nodes and training times

In fact, the training times of different network structures reflect the speed of convergence of different network structures. From the training times curve, it can be found that the number of required training increases exponentially with the increase of the number of hidden layer nodes. Therefore, it is necessary to weigh the choice of the topology of the BP network rather than the minimization of the mean square of the experimental results.

\subsection{Experimental result analysis}

According to the classification error and convergence speed of BP network composed of different hidden layer nodes, it can be found that when the number of hidden nodes is 24 , and the network MSE basically reaches the minimum value. At this point, in order to achieve the goal, the network accuracy training times is 11710 . By contrast, it can be found that the increase of the number of hidden layer nodes is more than 24, and the network performance has not been significantly improved, but the convergence speed has obviously slowed down. Therefore, in the Internet of things, the BP network topology used in the classification experiment of the data set is: 10 input layer nodes, 24 hidden layer nodes, and 6 output layer nodes. Finally, if the error is less than 0.5 , then it is the correct classification result. The statistical classification results are shown in Table 2. The results show that the final classification accuracy is as high as $98 \%$. 
Paper-A Data Classification Algorithm of Internet of Things Based on Neural Network

Table 2. Comparison of network learning rate and training frequency

\begin{tabular}{|c|l|c|c|c|c|}
\hline $\begin{array}{c}\text { Experimental data } \\
\text { set }\end{array}$ & $\begin{array}{c}\text { Classification } \\
\text { type }\end{array}$ & Training set & Test set & $\begin{array}{c}\text { The number of } \\
\text { the correct } \\
\text { classification }\end{array}$ & $\begin{array}{c}\text { Classification } \\
\text { accuracy }\end{array}$ \\
\hline \multirow{2}{*}{ KJ2010D110K } & $\begin{array}{l}\text { First classifica- } \\
\text { tion result }\end{array}$ & 1000 & 1000 & 981 & $98.1 \%$ \\
\cline { 2 - 6 } & $\begin{array}{l}\text { Cross validation } \\
\text { results }\end{array}$ & 1000 & 1000 & 989 & $98.9 \%$ \\
\hline
\end{tabular}

In the whole classification experiment, the time consumed by the single machine executing 11710 times of BP network training is about $257 \mathrm{~s}$. The time that spent in classifying the 1000 sets of test sets is less than $1 \mathrm{~s}$.

\section{Conclusion}

In order to verify the effectiveness of the improved BP algorithm, this paper implements a data classification system in the Internet of Things. Through the system, a lot of classification experiments were carried out. The results show that the improved BP algorithm can accelerate the convergence speed of the network in a certain training interval. The BP neural network is used to classify the data set in the Internet of Things. The success rate of classification is as high as $98 \%$, that is, it can meet the requirement of general data classification. By analyzing the error surface of a single neuron, it is found that the network is not trapped in the local minimum. Obviously, the improved BP algorithm can improve the performance of BP neural network to a certain extent.

The introduction and development of the Internet of things has brought great opportunities and challenges to the existing Internet system. Faced with the increasingly mature clustering technology, many algorithms are expected to break through its computing bottlenecks. Although this paper puts forward the data classification experiment scheme under the Internet of Things system, there are still some problems that need further study. The Internet of things is too broad. A data classification scheme or model is difficult to apply to all situations. Especially in dealing with large amounts of high-dimensional data, the existing BP network algorithms need to be proved.

\section{References}

[1] Perera, C., Zaslavsky, A., Christen, P., \& Georgakopoulos, D. (2014). Context aware computing for the internet of things: A survey. IEEE Communications Surveys \& Tutorials, 16(1), 414-454. https://doi.org/10.1109/SURV.2013.042313.00197

[2] Khan, M. A., Khan, S., Shams, B., \& Lloret, J. (2016). Distributed flood attack detection mechanism using artificial neural network in wireless mesh networks. Security and Communication Networks, 9(15), 2715-2729. https://doi.org/10.1002/sec.1204

[3] Xu, L., \& Liu, S. (2013). Study of short-term water quality prediction model based on wavelet neural network. Mathematical and Computer Modelling, 58(3), 807-813. https://doi.org/10.1016/j.mcm.2012.12.023 
[4] Luo, X., Lv, Y., Zhou, M., Wang, W., \& Zhao, W. (2016). A laguerre neural networkbased ADP learning scheme with its application to tracking control in the Internet of Things. Personal and Ubiquitous Computing, 20(3), 361-372. https://doi.org/10.1007/s00779-016-0916-X

[5] Botta, A., De Donato, W., Persico, V., \& Pescapé, A. (2016). Integration of cloud computing and internet of things: a survey. Future Generation Computer Systems, 56, 684-700. https://doi.org/10.1016/j.future.2015.09.021

[6] Ren, C., An, N., Wang, J., Li, L., Hu, B., \& Shang, D. (2014). Optimal parameters selection for BP neural network based on particle swarm optimization: A case study of wind speed forecasting. Knowledge-Based Systems, 56, 226-239. https://doi.org/10.1016/j.kno sys.2013.11.015

[7] Yu, F., \& Xu, X. (2014). A short-term load forecasting model of natural gas based on optimized genetic algorithm and improved BP neural network. Applied Energy, 134, 102113. https://doi.org/10.1016/j.apenergy.2014.07.104

\section{$7 \quad$ Author}

Zhenjun Li is with Hunan Radio and TV University, Hunan, China (e-mail: 278060389@qq.com).

Article submitted 13 August 2017. Published as resubmitted by the author 18 September 2017. 